Kathrin Rutishauser

Für die Schweizerische Gesellschaft für Phytotherapie (SMGP) ist diese Schnittstelle Neuland. Mit der laufenden Gesetzesänderung des Heilmittelgesetzes (HMG 2), welche Chancen, aber auch Risiken für die Weiterentwicklung der Phytotherapie mit sich bringt, gewinnt das Thema aktuell an Bedeutung. Eine sinnvolle Abgrenzung von pflanzlichen Arzneimitteln gegenüber sowohl Nahrungsergänzungsmitteln als auch Medizinprodukten mit pflanzlichen Stoffen ist zwingend nötig. Bei den exklusiven Mittagshäppchen und Kaffeepausen im Kongresszentrum Trafo in Baden konnte das Thema weiter vertieft und «verdaut» werden.

\section{Pflanzliche Arzneimittel versus}

Nahrungsergänzungsmittel,

Medizinprodukte - nur eine regulatorische Fragestellung oder ein grosses Problem für die

Phytotherapie? (Dr. Klaus Peter Latté, Berlin)

Wann ist ein Produkt als Arzneimittel, als Medizinprodukt oder als Nahrungsergänzungsmittel einzustufen? Die Unterscheidung erfolgt aufgrund unterschiedlicher Zweckbestimmungen, welche gesetzlich geregelt sind. Pflanzliche Arzneimittel sollen Heilung, Linderung oder Verhütung von Krankheiten oder krankhaften Beschwerden ermöglichen. Nahrungsergänzungsmittel mit pflanzlichen Inhaltsstoffen dienen zur Ergänzung der normalen Ernährung; sie

\title{
31. Schweizerische Tagung für Phytotherapie: Phytotherapie und pflanzliche Nahrung: Partner oder Gegensatz?
}

Ernährung und pflanzlicher Nahrung im Speziellen wird heutzutage ein hoher Stellenwert zugeschrieben. Rund 350 Personen vermochte das diesjährige Thema der 31. Schweizerischen Jahrestagung für Phytotherapie, welches sich dem Spannungsfeld Phytotherapie und pflanzliche Nahrung widmete, anzulocken (Abb. 1).

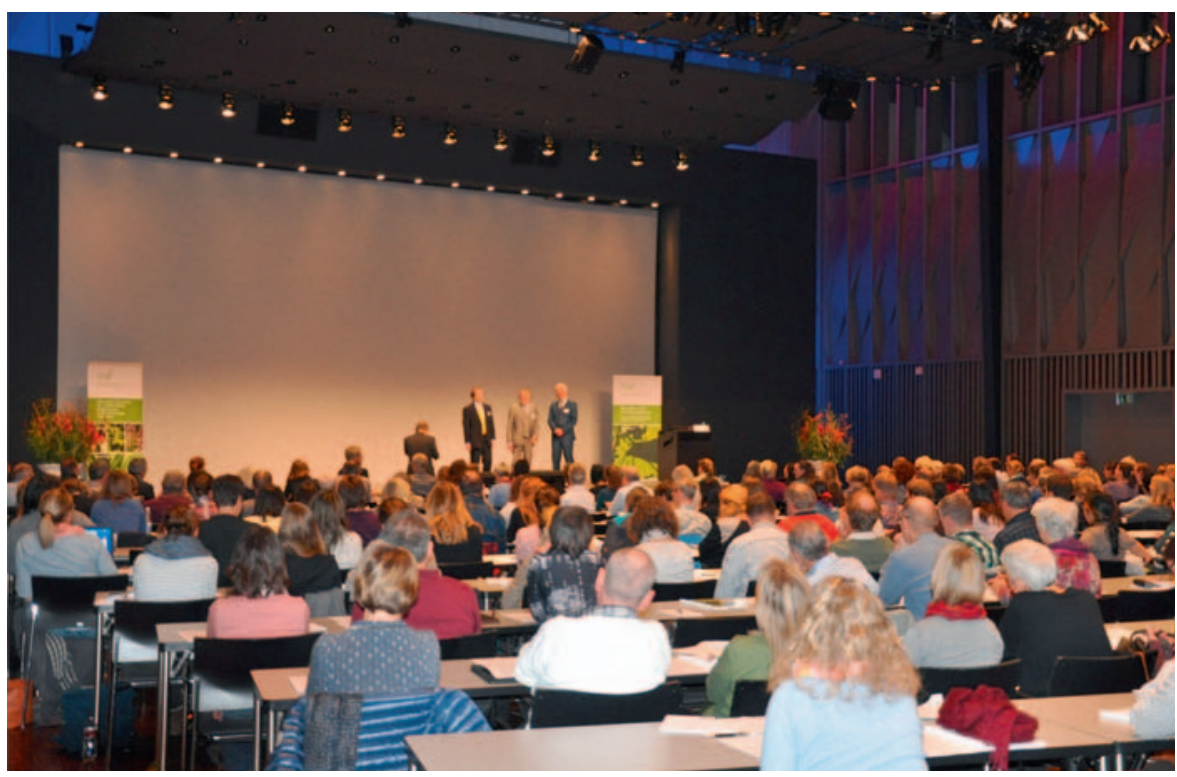

Abb. 1. Blick in den Tagungssaal im Trafo in Baden.

sind also nicht dazu bestimmt, Krankheiten zu heilen. Die Anforderungen für Arzneimittel und Medizinprodukte sind im HMG geregelt. Deren Umsetzung erfolgt für Arzneimittel unter anderem gemäss der Verordnung über die Arzneimittel (VAM) sowie der Verordnung des Schweizerischen Heilmittelinstituts über die vereinfachte Zulassung von Komplementärund Phytoarzneimitteln (KPAV), während Medizinprodukte der Medi- zinprodukteverordnung (MepV) unterliegen. Nahrungsergänzungsmittel sind im Lebensmittelgesetz geregelt (Tab. 1).

In der Revision des HMG wurden erweiterte Bestimmungen für die vereinfachte Zulassung von pflanzlichen Arzneimitteln und Arzneimitteln der Komplementärmedizin aufgenommen. So werden wohl in Zukunft bibliografische Nachweise zur Wirksamkeit und Sicherheit oder Anwendungsbe-

\section{KARGER}

(C) 2017 S. Karger GmbH, Freiburg 
Tab. 1. Vergleichende Gegenüberstellung der Produktkategorien Arzneimittel (AM), Medizinprodukte (MP) und Nahrungsergänzungsmittel (NEM) im Hinblick auf pflanzliche Stoffe (Quelle: Tagungsband 31. Schweizerische Jahrestagung für Phytotherapie, Dr. Klaus Peter Latté)

\begin{tabular}{|c|c|c|c|}
\hline & \multicolumn{2}{|l|}{ Heilmittel } & \multirow[t]{2}{*}{ Nahrungsergänzungsmittel } \\
\hline & Arzneimittel & Medizinprodukt & \\
\hline Wichtigste gesetzliche Grundlage & Neu: HMG2 & $\mathrm{HMG}+\mathrm{MepV}$ & Rev. LMG + VNem \\
\hline Zielgruppe & $\begin{array}{l}\text { Mensch (Patienten) } \\
\text { und Tier }\end{array}$ & Mensch (Patient / Anwender) & für generelle Bevölkerung \\
\hline Zweck & $\begin{array}{l}\text { medizinische Einwirkung: } \\
\text { Erkennung, Verhütung } \\
\text { oder Behandlung von } \\
\text { Krankheiten, Verletzungen } \\
\text { und Behinderungen }\end{array}$ & medizinische Verwendung & $\begin{array}{l}\text { Ergänzung der normalen } \\
\text { Ernährung }\end{array}$ \\
\hline Wirkmechanismus & $\begin{array}{l}\text { pharmakologisch, } \\
\text { metabolisch, } \\
\text { immunologisch }\end{array}$ & $\begin{array}{l}\text { nicht pharmakologisch, } \\
\text { metabolisch, } \\
\text { immunologisch }\end{array}$ & $\begin{array}{l}\text { ernährungsspezifische } \\
\text { oder physiologische } \\
\text { Wirkungen }\end{array}$ \\
\hline $\begin{array}{l}\text { Inverkehrbringen pflanzlicher } \\
\text { Stoffe }\end{array}$ & $\begin{array}{l}\text { vereinfachtes Verfahren } \\
\text { unter anderem für } \\
\text { Phytoarzneimittel }\end{array}$ & $\begin{array}{l}\text { nach Nachweis der grund- } \\
\text { legenden Anforderungen, } \\
\text { Konformitätsbewertungs- } \\
\text { verfahren, Meldepflicht } \\
\text { bei Inverkehrbringen }\end{array}$ & $\begin{array}{l}\text { Anhang II zu VNem } \\
\text { (Negativliste); } \\
\text { Meldepflicht bei BLV }\end{array}$ \\
\hline Kosten und Zeit & sehr hoch, Jahre & mässig hoch & gering \\
\hline Produktsicherheit, Qualität & sehr hoch (Zulassung!) & $\begin{array}{l}\text { hoch (Konformitäts- } \\
\text { bewertungsverfahren) }\end{array}$ & LM müssen sicher sein \\
\hline Behördlicher Vollzug & $\begin{array}{l}\text { stark, } \\
\text { hohe Regulierungsdichte }\end{array}$ & $\begin{array}{l}\text { mässig, } \\
\text { weniger Vollzugsregeln }\end{array}$ & $\begin{array}{l}\text { mässig, } \\
\text { weniger Vollzugsregeln }\end{array}$ \\
\hline Forschung und Produktinnovation & hoch? & (hoch) & hoch? \\
\hline $\begin{array}{l}\text { Erstattungsfähigkeit durch Grund-/ } \\
\text { Privatversicherung }\end{array}$ & gut dokumentierte & ilmittel: erstattungsfähig & nein \\
\hline
\end{tabular}

lege ausreichen. Gleichzeitig gibt es auch bei den Nahrungsergänzungsmitteln Änderungen im Rahmen der umfassenden Revision der Schweizer Lebensmittelgesetzgebung («LARGO»). Diese hat die Anpassung an das EURecht, den Abbau von Handelshemmnissen und die einheitliche Risikobewertung zum Ziel. Im bisherigen Lebensmittelrecht galt das Positivgesetz, d.h., was nicht ausdrücklich erlaubt ist, ist verboten. In Harmonisierung mit den EU-Regelungen werden in der Schweiz neu alle Lebensmittel erlaubt sein, wenn sie sicher sind und den Vorgaben entsprechen. Bestandteile von Nahrungsergänzungsmitteln sind nach wie vor Vitamine, Mineralstoffe und sonstige Stoffe, die neu in einer eigenen Verordnung (VNem) und deren Anhängen I und II («Liste der Pflanzen, Pflanzenteile und daraus her- gestellten Zubereitungen, deren Verwendung in Nahrungsergänzungsmitteln nicht zulässig ist») enthalten sind. Hier wird offensichtlich, dass eine klare Abgrenzung zwischen den Produktgruppen mit pflanzlichen Stoffen notwendig ist. Bis anhin wurde die Zuordnung Arzneimittel und Nahrungsergänzungsmittel mit pflanzlichen Stoffen in die jeweilige Produktkategorie mithilfe einer Liste von Swissmedic und dem Bundesamt für Gesundheit (BAG) vorgenommen. Die neue Liste in Anhang II der VNem ist nicht deckungsgleich mit der bestehenden Swissmedic/BAG-Liste (z.B. sind Ginkgo biloba und Valeriana officinalis nicht in Anhang II der VNem als verbotene Stoffe in Nahrungsergänzungsmitteln aufgeführt). In Nahrungsergänzungsmitteln müssen Pflanzenextrakte und deren wirksame Be- standteile analog zu den Lebensmitteln «nur» mengenmässig in der Nährwertdeklaration aufgeführt werden. Für pflanzliche Arzneimittel bestehen in der Phytoanleitung viel umfassendere Deklarationsvorschriften. Für eine klare Abgrenzung insbesondere bei «Dual-use-Stoffen», welche in Abhängigkeit von der Dosis und Anwendung sowohl als Lebensmittel als auch als Arzneimittel eingesetzt werden können, fehlen in Anhang II der VNem unter anderem Vorgaben zur näheren Charakterisierung (z.B. Droge-Extrakt-Verhältnis), Darreichungsform und Dosierung der Produkte.

Sowohl das HMG als auch die Revision des Lebensmittelrechts sehen Änderungen vor, welche für die Weiterentwicklung der Phytotherapie genutzt werden können. Das HMG fördert mit dem vereinfachten Zulas- 


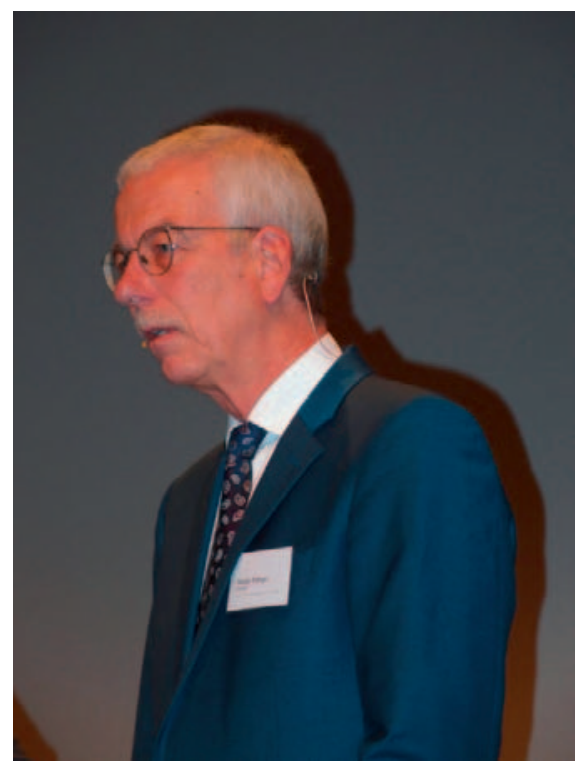

Abb. 2. "Antientzündliche Wirkungen von Ernährung und Phytotherapie» stellten den Fokus des Referats von Dr. med. Rainer Stange dar.

sungsverfahren für pflanzliche Arzneimittel einen schnelleren Markteintritt und Produktinnovationen bei bekannt hoher Qualität, während die Revision des Lebensmittelgesetzes vermutlich einen Anreiz für Innovationen im Bereich pflanzlicher Nahrungsergänzungsmittel zur Folge haben wird. Da sich viele Konsumenten von Nahrungsergänzungsmitteln viel versprechen, ist eine sinnvolle Abgrenzung aus Sicht der Phytotherapie zwingend notwendig. Fachgesellschaften wie der SMGP (Kasten) wird empfohlen, sich in einem Gremium für Abgrenzungsfragen, wie dies im nahen Ausland auch bereits gemacht wird, mit ihrem Wissen zu engagieren und aufzuzeigen, wo die Wirkungen und Potenziale von pflanzlichen $\mathrm{Zu}$ bereitungen liegen und wo eher nicht. Differenzieren ist in der Phytotherapie nichts Neues: Pflanzliche Arzneimittel sind aufgrund ihrer unterschiedlichen Herstellung, Dosierung oder Darreichungsform in ihrer Wirkung oder ihrem Wirkungsspektrum nicht immer direkt vergleichbar. Dies ist bei pflanzlichen Nahrungsergänzungsmitteln sehr ähnlich.

Die 32. Schweizerische Jahrestagung für Phytotherapie wird am 23. November 2017 zum Thema «Phytotherapie in der Gynäkologie: Aus der Praxis - für die Praxis» stattfinden. Informationen dazu sowie zur organisierenden Gesellschaft, der SMGP, sowie zum Fähigkeitsprogramm Phytotherapie für Ärztinnen und Ärzte sowie Apotheker und Apothekerinnen (und in Veterinärmedizin) gibt es auf der Website: www.smgp.ch. Die SMGP ist der einzige Verband in der Schweiz, der eine kontinuierliche Fortund Weiterbildung in Phytotherapie für die akademischen Medizinalberufe anbietet.

Antientzündliche Wirkungen von Ernährung und Phytotherapie - sind Synergismen denkbar? (Dr. med. Rainer Stange, Berlin) (Abb. 2)

Das metabolische Syndrom wird als entscheidender Risikofaktor für eine Erkrankung der arteriellen Gefässe bezeichnet und gewinnt immer mehr an Bedeutung. Neben genetischen Faktoren gilt der Lebensstil, welcher auch Bewegung und Ernährung umfasst, als schützend und begünstigend für die Entwicklung und den Verlauf des metabolischen Syndroms. Diverse Pflanzen, insbesondere deren sekundäre Inhaltsstoffe, haben einen günstigen Einfluss auf akute und chronische Entzündungen. Studien erfordern grosse Patientenzahlen und lange Therapiezeiträume, sodass es erst wenig gesicherte Resultate gibt. Sowohl Carotinoide, welche für die rote Färbung von z.B. Tomaten und Wassermelonen verantwortlich sind, als auch Anthocyane, welche die violette/dunkelrote Färbung von Trauben und Beeren veranlassen, haben entzündungshemmende Eigenschaften. Bei chronisch entzündlichen Gelenkerkrankungen wurde z.B. Sonnenhut (Echinacea spp.) als entzündungshemmende Pflanze (COX-Hemmung) identifiziert. Auch Sojabohnen-Extrakte konnten mit entzündungshemmenden Effekten auf Basis eines Projektes, das aus der Weltliteratur die Beeinflussung des metabolischen Syndroms durch pflanzliche Lebensmittel und daraus hergestellten Extrakten ableitete, epidemiologisch assoziiert werden. Dasselbe gilt für Traubenkerne und Schwarztee. Die wichtigsten Resultate von sekundären Pflanzenstoffen mit gesicherter Bedeutung liefern die Omega-6-Fettsäuren (proinflammatorisch) und
Omega-3-Fettsäuren (antiinflammatorisch). Der bekannteste Vertreter der aus pflanzlicher Quelle stammenden Omega-3-Fettsäure ist die a-Linolensäure, welche unter anderem in Leinsamen und in Leinöl reichlich vorkommt. Als Alternative für die tierische Omega-3-Fettsäure aus fettreichem Fisch in Kapselform gibt es auch eine vegane Antwort: Omega3-Fettsäuren aus Algen.

\section{Die Bedeutung der ungesättigten} Fettsäuren für Tier und Mensch (Dr. Florian Leiber, Frick)

Ein weiteres Nahrungsmittel, welches sich heute als wertvolle Quelle für Omega-3-Fettsäure herausgestellt hat, war bereits 1931 bekannt: die Butter aus dem Lötschental. Als der amerikanische Zahnarzt und Ernährungswissenschaftler Weston Price die Schweiz bereiste, war ihm aufgefallen, dass die Kinder aus dem Lötschental im Vergleich zu St. Galler Stadtkindern überdurchschnittlich gesund waren. Das auch von den Lötschentalern selbst heilig verehrte Produkt (es wurde auch $\mathrm{zu}$ religiösen Zwecken verbrannt), enthielt bis zu dreimal mehr wertvolle Omega-3-Fettsäuren als Butter aus konventionell produzierter Milch. Dies geht auf den Weidegang und die auf den Alpweiden zahlreich vorhandenen Kräuter zurück (statt Mais und Getreide). Blattreiche Diäten sind grundsätzlich reicher an Omega-3-Fettsäuren, da letztere im grünen Blattgewebe im Zusammenhang mit der Photosynthese entstehen. Omega-6-Fettsäuren hingegen werden eher in Samen als Speicheröle eingelagert. Essenziell für alle Säuger ist die Aufnahme beider Fettsäuren, da deren Synthese aufgrund fehlender 
Enzyme nicht möglich ist. Eine gute Balance ist wichtig, weil beide Fettsäuren um dieselben Enzyme im Stoffwechsel konkurrieren. Da die Konzentration von Omega-3-Fettsäuren in Milch und Fleisch vom Anteil an Kräutern und Getreide in der Ernährung der Tiere abhängt (und weniger von der geografischen Lage der Haltung), zeichnet sich ein grosses Potenzial von Produkten terrestrischer Herbivoren aus krautreichen Weiden unserer Alpen ab. Eine Umstellung des Humankonsums auf Milchprodukte aus solcher Produktion könnte die Omega-3-Aufnahme einer erwachsenen Person mit durchschnittlicher Konsumgewohnheit theoretisch um $43 \%$ steigern. Das würde gleichzeitig der Überfischung der Meere entgegenwirken, denn dies entspricht dem täglichen Verzehr von 800 g Lachs.

Eine Umstellung des Produktionssystems («alpine» Produktion) mit einem hohen Anteil an Kräutern in der Ernährung der Milchkühe wäre eine «Win-Win-Win»-Situation für die Tiere, die menschliche Ernährung und auch für die Weltmeere und deren Biodiversität.

\section{Einfluss von pflanzlicher Nahrung}

auf die Neurophysiologie des Essens (Dr. Rosmarie Clara, Prof. Dr.

Wolfgang Langhans, Zürich)

Mit der weltweiten Adipositas-Epidemie sind ernährungsbedingte Erkrankungen in der öffentlichen Wahrnehmung stärker in den Fokus geraten. Durch Übergewicht und dadurch begünstigte Gesundheitsprobleme wie Typ-2-Diabetes, kardiovaskuläre Erkrankungen und Gelenkprobleme steht auch unser Gesundheitssystem vor neuen Herausforderungen. So ist gesunde Ernährung neben regelmässiger körperlicher Aktivität wichtig für die Prävention von nicht übertragbaren Krankheiten.

Der Nahrungsaufnahme liegen komplexe Feedback-Signale (physiologischer Regelkreis) zugrunde. Obwohl fast alle Säuger eine angeborene
Präferenz für Süsses haben und Bitteres ablehnen, ist die Nahrungsauswahl primär über erlernte Präferenzen und Aversionen gesteuert. Der eigentlich effiziente physiologische Regelkreis führt in einer Umwelt, wo konstant attraktive Nahrung vorhanden ist, zu einer überhöhten Nahrungsaufnahme. Dies, weil es im Verlauf der Evolution entscheidend war, ein plötzliches Angebot attraktiver Nahrung maximal zu nutzen.

Unsere Ernährung hat sowohl einen direkten als auch einen indirekten Einfluss auf Stoffwechsel und Verdauung, indem Inhaltsstoffe der Ernährung die Nahrungsaufnahme und somit das Körpergewicht modulieren können. Ein Beispiel hierfür sind die Gewürze, welche einen direkten positiven Effekt auf die Gesundheit haben (z.B. Steigerung der Aktivität von Leber und Galle, Förderung der Verdauung und der Aquarese, Entzündungshemmung) sowie gleichzeitig das Essen schmackhafter machen und somit die Nahrungsaufnahme stimulieren. Andere Substanzen wie komplexe Kohlenhydrate können die Nahrungsaufnahme hemmen, indem sie die gastrointestinale Passage verzögern und den Anstieg des Blutzuckers reduzieren. Neuere Untersuchungen mit Thylakoiden (d.h. Bestandteilen von Chloroplasten) zeigten, dass damit die Nahrungsaufnahme nennenswert gehemmt werden kann. Die Mechanismen der gesundheitsfördernden Effekte von pflanzlichen Nahrungsbestandteilen sind jedoch noch nicht im Detail bekannt.

Antioxidantien und Gesundheit:

Ein Reagenzglaseffekt oder mehr?

(PD Dr. med. Gudrun Ulrich-

Merzenich, Bonn) (Abb. 3)

Antioxidantien schützen andere Stoffe vor Oxidation und sind massgeblich bei der Erhaltung der RedoxHomöostase beteiligt. Bei erhöhtem oxidativen Stress werden reaktive Sauerstoffspezies (ROS) gebildet, welche physiologische Abläufe der Zellen

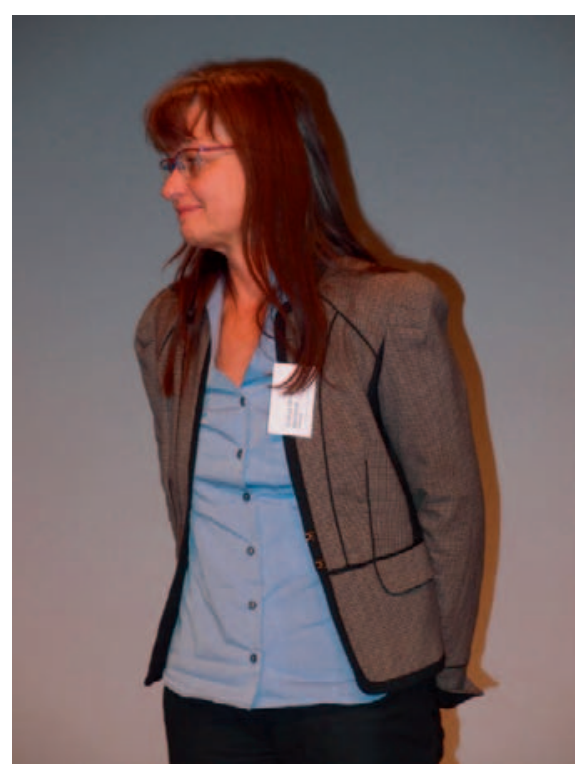

Abb. 3. PD Dr. med. Gudrun Ulrich-Merzenich widmete sich dem Thema «Antioxidantien und Gesundheit: Ein Reagenzglaseffekt oder mehr?».

schädigen und z.B. Arteriosklerose oder den Alterungsprozess fördern können. Die Antioxidantien zeigen, zumindest im Reagenzglas, einen schützenden Effekt. Dieser Effekt beruht sowohl auf der Aktivierung der MAP-Kinasen als auch auf der Beeinflussung der Genexpression als Signalmoleküle. Verschiedene Meta-Analysen belegen aber auch, dass die Einnahme von Antioxidantien in Form von Supplementen nicht immer die erwartete Wirkung zeigt und höhere Dosen von $\beta$-Carotin, Vitamin A und Vitamin E sogar mit einer höheren Mortalitätsrate verbunden sind. Auch zur Prävention kardiovaskulärer Erkrankungen mit Vitaminen liegen widersprüchliche Resultate vor. Unter anderem wird in der Literatur argumentiert, dass nicht die antioxidativ wirkenden Vitamine, sondern die pflanzlichen Polyphenole die protektive Wirkung hinsichtlich koronarer Herzerkrankungen ausüben. Deshalb hat die Referentin betont, dass eine Supplementierung eine abwechslungsreiche, an Antioxidantien reiche Ernährung nicht ersetzen kann. Eine pflanzliche Ernährung führt im Vergleich zu einer auf tierischen Nahrungsmitteln basierten Ernährung 
grundsätzlich $\mathrm{zu}$ einer vermehrten Aufnahme an Antioxidantien. Zwischen der Aufnahme von Antioxidantien und deren antioxidativer Aktivität in unseren Körperzellen gibt es keinen direkten Zusammenhang. Dies hängt wohl mit der Bioverfügbarkeit zusammen, welche durch verschiedenste Aspekte wie Zubereitung, Lebensmittelmatrix, Absorption und Resorption sowie dem Metabolismus sowie anabolen und katabolen Reaktionen beeinflusst wird.

Zusammenfassend zeigte das Referat, dass eine Supplementierung je nach Ernährungssituation vernünftig sein kann - beispielsweise, wenn ein Mangel vorliegt. Bei einer Supplementierung bei normaler Ernährung und «gesättigten Werten» ist im Speziellen bei den fettlöslichen Vitaminen A und E sowie $\beta$-Carotin Vorsicht geboten. Es sind jedoch noch weitere Untersuchungen nötig, um das Zusammenspiel von Antioxidantien und ROS besser zu verstehen.

\section{Knoblauch - was ist von den}

Hypes geblieben? (Prof. Dr. med.

Reinhard Saller, Zürich)

Der Referent verwies auf das Schicksal des Knoblauchs, der noch in den 1990er-Jahren hoch gepriesen wurde. Knoblauch ist eine der ältesten und gut dokumentierten Arznei- und Gewürzpflanzen und auch in der Schweiz seit Längerem gut belegt. Traditionell wird er bei Verdauungsstörungen, Erkältungskrankheiten und Asthma bronchiale sowie als Stärkungsmittel eingesetzt. Verschiedene weitere Anwendungsgebiete sind ab Ende des 19. Jahrhunderts dazugekommen, so z.B. die Prophylaxe bei Erkrankungen im Alter (wie allgemeine Schwäche) oder zur leichten Senkung von erhöhten Blutfettwerten und Blutdruck. Äusserliche Anwendungen sind unter anderem bei Hautkrankheiten und Pilzinfektionen bekannt. Der «Knoblauch-Hype» der 1990er-Jahre hing grösstenteils mit der Verkündung von Knoblauch als «natürlichem» Cholesterin- und
Blutdrucksenker zusammen. Diese Effekte waren jedoch nicht besonders ausgeprägt, was wahrscheinlich dazu beitrug, dass der Knoblauch-Hype rasant abflachte.

In letzter Zeit werden knoblauchhaltige Präparate vermehrt wieder als pflanzliche Vielstoffgemische mit einem breiten Anwendungsspektrum, guter Verträglichkeit und geringem Interaktionspotenzial betrachtet. So konnten neuere Studien nicht nur eine signifikante Reduktion des Blutdrucks, sondern auch den positiven Einfluss von Knoblauchpulver auf verschiedene kardiovaskuläre Risikofaktoren nachweisen. Anwendungsbereiche könnten sich auch in der Prävention/Therapie einiger Tumorerkrankungen ergeben. Ein umfassender Übersichtsartikel (Review) zeigte bei vermehrter Knoblaucheinnahme ein verringertes Risiko für Prostatatumoren. Hört man all dies, würde das Aufkommen eines nächsten Knoblauch-Hypes nicht erstaunen ...

Personalisierte Ernährung -

Nutrigenomik am Beispiel von

Arteriosklerose und Diabetes

(Dr. Guy Vergères, Bern)

Personalisierte Ernährung tönt vielversprechend und ist in aller Munde. Doch was versteht man genau darunter? Werden wir in Zukunft im Restaurant eine auf unsere Gene abgestimmte Menü-Auswahl erhalten? Ein eindrückliches Beispiel, wie die Umwelt mit den Genen interagiert, hat der Referent anhand der Pima-Indianer aufgezeigt. Die Pima-Indianer, welche in Arizona mit dem westlichen Überangebot von energiedichter und somit attraktiver Nahrung leben, weisen eine extrem hohe Prävalenz an Typ2 -Diabetes auf $(>50 \%)$. Bei den PimaIndianern in Mexiko hingegen ist Diabetes bei weniger als $10 \%$ ein Problem. Die hohe Rate lässt auf die Exposition ihres Genoms schliessen, welches in der Vergangenheit Zeiten mit Nahrungsmittelrestriktionen ausgesetzt war und heute nicht mit der ubiquitären Verfügbarkeit energiedichter Nahrung umgehen kann. Ein weiteres Beispiel einer genetischen «Fehlanpassung» an das heutige Nahrungsmittelangebot (oder die noch laufende Evolution) ist die vererbbare Fruktose-Intoleranz. Wenige Menschen verfügen über eine Genvariante eines Enzyms (Aldolase B) mit geringer Aktivität, welches an der Verstoffwechselung von Fruktose beteiligt ist. Erst mit erhöhter Fruktose-Aufnahme durch industriell hergestellte Produkte wurde diese Genvariante zu einem Problem: Die Akkumulierung von Fruktose-1-Phosphat im Gewebe führt zu Hypoglykämie und Folgeschädigungen. In der wissenschaftlichen Literatur sind viele solche Interaktionen zwischen Genen und spezifischen Nährstoffen beschrieben. Einige Firmen versuchen darauf basierend kommerzielle Gentests zu verkaufen. Kaffee-Junkies können so herausfinden, ob sie Träger einer schwach aktiven Genvariante von CYP1A2, dem Hauptenzym beim Koffein-Metabolismus, sind. Langsame Metabolisierer, die 2-3 Tassen Kaffee pro Tag tranken, hatten ein höheres Risiko, einen Herzinfarkt zu erleiden, als Menschen mit derselben Genvariante, die aber nur eine Tasse Kaffee pro Tag tranken. Obwohl die wissenschaftliche Qualität solcher Studien grösstenteils anerkannt ist, stellen sich auf der klinischen Seite noch diverse Fragen. Nur wenige Gene (Laktase-Gen) zeigen sich auch eindeutig im Phänotyp (Laktose-Intoleranz). Viele chronische Erkrankungen sind jedoch polygen bedingt und können nicht auf ein einzelnes Gen zurückgeführt werden. So ist offensichtlich, dass die heute verfügbaren Gentests schnell an ihre Grenzen stossen.

Verschiedene chemische Modifikationen, insbesondere epigenetische Modifikationen in Form von DNAMethylierungen, werden durch die Umwelt beeinflusst und haben Auswirkungen auf die phänotypische Aktivität der Zellen. Epigenetische Modifikationen können auch über 


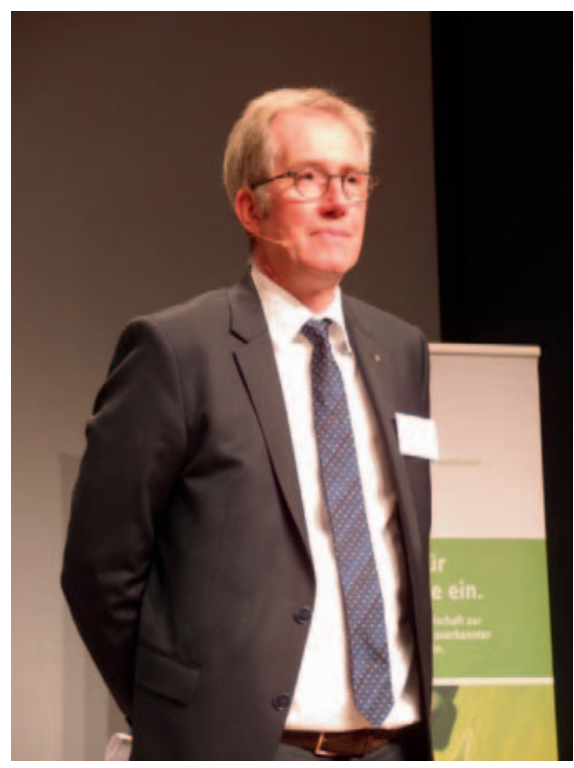

Abb. 4. Dr. Andreas Biller stellte in seinem Vortrag die Frage: «Curcuma - ein Arzneimittel ohne Chance auf Zulassung?»

Generationen weitervererbt werden. Verschiedene Projekte, unter anderem das europäische Projekt «Food$4 \mathrm{Me}$ », untersucht die Beziehung zwischen Nahrung und Genexpression. Nicht nur in der Ernährung, sondern auch in der Medizin könnte eine Personalisierung nötig und möglich sein. Dies wurde vor allem klar, als der Referent aufgezeigte, dass gemäss einem kürzlich in der Zeitschrift Nature erschienenen Artikel die Top-10-Medikamente in den USA im schlechtesten Fall bei einer von 25 Personen und im besten Fall bei einer von 4 Personen wirken.

Die Personalisierung von Ernährung und Medikation zeigt also Chancen auf, birgt aber auch Risiken in sich, wie z.B. ethische Fragen, die allerdings nicht Thema des Referats waren.

\section{Curcuma - ein Arzneimittel}

ohne Chance auf Zulassung?

(Dr. Andreas Biller, Winsen) (Abb. 4)

In der HMPC-Monographie für Gelbwurz (Curcuma longa) wird einzig auf die Indikation bei Verdauungsproblemen hingewiesen. Dies ist jedoch nur eine von vielen Anwendungen der Gelbwurz, die zur Familie der
Ingwergewächse gehört. Insbesondere für Darmerkrankungen ist eine hohe Wirksamkeit nachgewiesen. So wird Curcumin, der Hauptinhaltsstoff der Gelbwurz, aktuell in den deutschen Leitlinien für Ärzte zur adjuvanten Therapie von Colitis ulcerosa empfohlen. Curcumin verstärkt das antioxidative und antiinflammatorische System und hemmt gleichzeitig die Bildung von proinflammatorischen Enzymen.

Eine grosse Herausforderung stellt bis heute die Bioverfügbarkeit dar, denn Curcumin ist stark hydrophob, was eine geringe Resorption aus den Verdauungsorganen zur Folge hat. Eine markante Verbesserung der Bioverfügbarkeit konnte z.B. in Kombination mit Piperin aus Schwarzem Pfeffer nachgewiesen werden. Der Pfeffer hemmt die hepatische und intestinale Verbindung von Curcumin mit Glucuronsäure. Eine weitere Möglichkeit, die Bioverfügbarkeit zu verbessern, besteht darin, Curcumin in Micellenform zu verabreichen. Dabei wird Curcumin im lipophilen Innenraum von einer hydrophilen Hülle eingeschlossen (Micelle), um dann dank der hydrophilen Oberfläche von der Darmwand besser aufgenommen werden zu können. Mit dieser Darreichungsform konnte im Vergleich zu pulverisierten Kurkuma-Extrakten eine 185-fach verbesserte Bioverfügbarkeit erreicht werden. Somit wird auch die Behandlung systemischer, entzündlicher Erkrankungen realistischer. Berichtet wird vom erfolgreichen Einsatz bei chronischen Entzündungen wie Arthrose und Arthritis.

Ähnlich wie beim Knoblauch ist das Wirkungspotenzial von Curcuma noch längst nicht ausgeschöpft.

\section{Fazit}

Die 31. Schweizerische Jahrestagung für Phytotherapie hat gezeigt, dass die Übergänge zwischen $\mathrm{Nah}$ rungspflanzen und Arzneipflanzen oft fliessend sind. Deshalb ist es nahelie- gend, dass sekundäre Pflanzenstoffe aus Ernährung und Phytotherapie sich in ihren Wirkungen ergänzen können. Die regulatorische Abgrenzung stellt eine Herausforderung dar; insbesondere stellt sich die Frage, wie sinnvoll es ist, wenn Nahrungsergänzungsmittel Wirksamkeitsansprüche der Phytotherapie übernehmen. Klar ist, dass beide Produktgruppen dem Erhalt der Gesundheit dienen sollen. In diesem Sinne kann die im Tagungsthema gestellte Frage «Phytotherapie und pflanzliche Ernährung: Partner oder Gegensatz?» mit einem abschliessenden Zitat von Hippokrates beantwortet werden: «Eure Nahrungsmittel sollen Eure Heilmittel und Eure Heilmittel Eure Nahrungsmittel sein!»

\section{Dank}

Wir danken den Referierenden, dass sie ihre Vortragskurzfassungen als Basis für diesen Bericht zur Verfügung gestellt haben. Eine diesbezügliche Dokumentation zur Tagung ist im Internet zu finden: www.sspm.org/ smgp/homeindex/jahrestagung.html.

\section{Korrespondenzadresse}

Prof. Dr. sc. nat. Beat Meier Geschäftsstelle SMGP c/o Zürcher Hochschule für angewandte Wissenschaften Fachgruppe Phytopharmazie und Naturstoffe Grüental, Postfach 335 8820 Wädenswil, Schweiz

Beat.Meier@zhaw.ch 